Document downloaded from:

http://hdl.handle.net/10251/56242

This paper must be cited as:

López Sánchez, J.; Romero Ruescas, C.; Gómez Barquero, D.; Cardona Marcet, N. (2011). On Transmitting of Global and Local Contents within DVB-T Single Frequency Networks. IEEE Latin America Transactions. 9(5):444-453. doi:10.1109/TLA.2011.6030975.

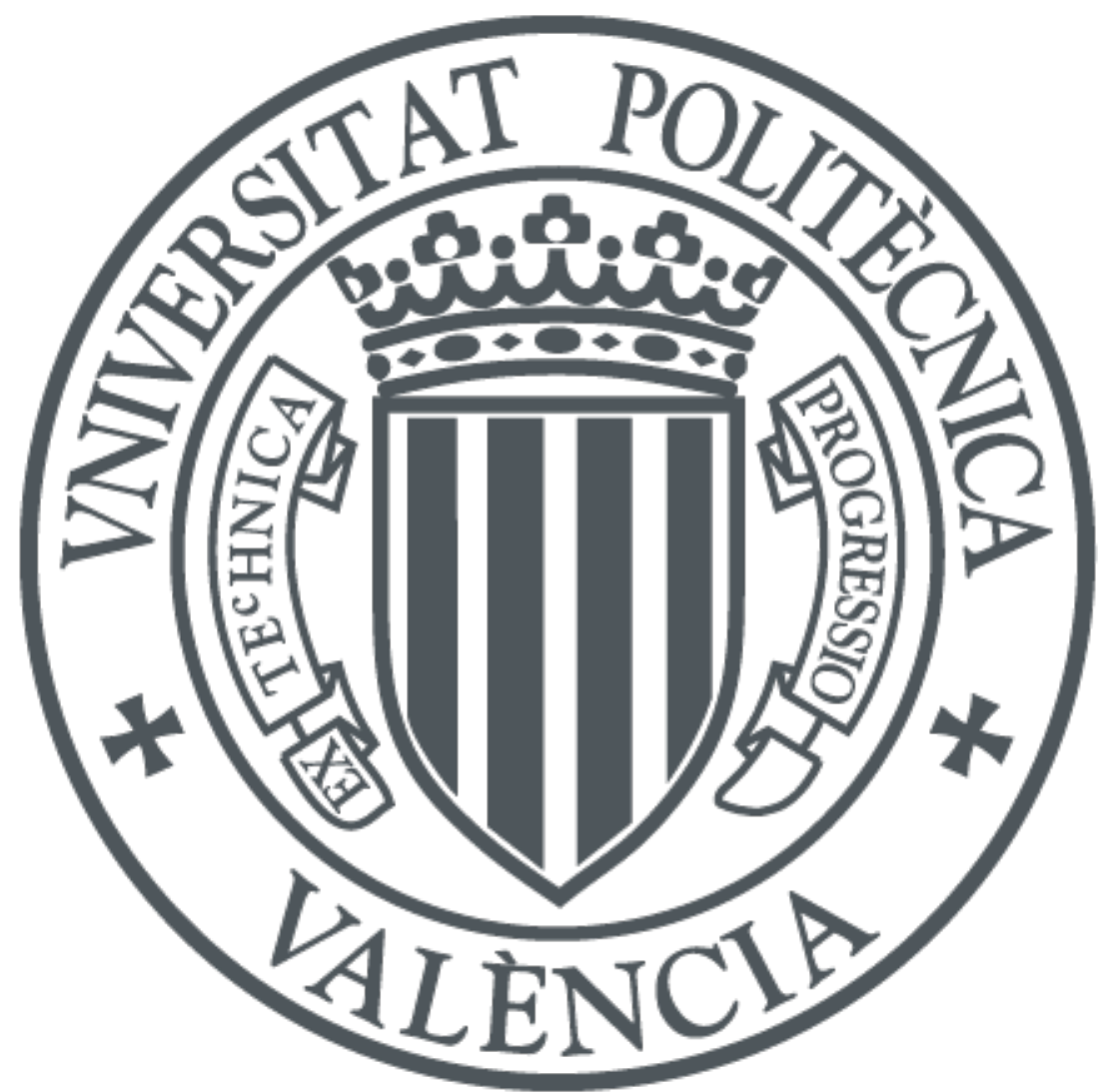

The final publication is available at

http://dx.doi.org/10.1109/TLA.2011.5993732

Copyright Institute of Electrical and Electronics Engineers (IEEE)

Additional Information 


\title{
On Transmitting of Global and Local Contents within DVB-T Single Frequency Networks
}

\author{
J. L. Sánchez, C. R. Ruescas, D. G. Barquero and N. Cardona
}

\begin{abstract}
Current broadcast digital networks make use of either a Single Frequency Network (SFN) or a Multi Frequency Network (MFN). None of these networks topologies is ideally suited for delivery of both global and local services in an efficiently way. MFNs enable the transmission of local services but need a significant amount of frequencies and therefore large amounts of valuable spectrum. SFNs are especially suitable for global services due to mutual support of the signals from multiples transmitters operated in the same frequency. SFNs are very spectrum efficiently. However they do not support local services. In this paper a hierarchical modulation method and Time Slicing technology are proposed for providing local and global contents in a terrestrial SFN (such DVB-T) keeping all its advantages. The hierarchical modulation has already been included in the DVB-T standard and time slicing solution is backwards compatible, in the sense that receivers that have been deployed in the original system can continue receiving data without hardware modifications. The performances of these technical solutions for both global and local services are analyzed. The applicability of these concepts is shown by a signal propagation simulation and coverage estimation in real scenario of Valencia region (Spain).
\end{abstract}

Keywords - DVB-T, Hierarchical Modulation, Local Contents, Time Slicing technology.

\section{INTRODUCCIÓN}

$\mathrm{E}$ $\mathrm{N}$ LAS actuales redes de difusión de televisión se transmiten distintos tipos de servicios, en función de la zona de la red a la que están dirigidos. Algunos servicios son de interés en toda la red, como por ejemplo los canales de televisión nacionales, que son transmitidos en el país entero, estos son los llamados servicios globales y su zona de cobertura es conocida como Área de Cobertura Global (GSA). Otros servicios, llamados locales, sólo son interesantes recibirlos en zonas geográficas delimitadas, como una ciudad por ejemplo. La zona donde tenemos servicios locales se llama área de servicios locales (en adelante LSA).

La televisión digital terrestre (TDT), presenta ventajas en la recepción móvil y en la difusión de contenidos regionales o locales en comparación con las transmisiones mediante cable o

Este trabajo ha sido cofinanciado por el Ministerio de Industria, Turismo y Comercio dentro del Plan Nacional de Investigación Científica, Desarrollo e Innovación Tecnológica 2008-2011 y el Fondo Europeo de Desarrollo Regional (FEDER) a través del proyecto tractor Redes Híbridas TSI-0203072010-165.

J. L. Sánchez, Universidad Politécnica de Valencia, Valencia, España, jailosan@iteam.upv.es

C. R. Ruescas, Universidad Politécnica de Valencia, Valencia, España, carrorue@iteam.upv.es

D. G. Barquero, Universidad Politécnica de Valencia, Valencia, España, dagobar@iteam.upv.es

N. Cardona, Universidad Politécnica de Valencia, Valencia, España, ncardona@dcom.upv.es vía satélite. Los contenidos o servicios locales pueden recibirse mediante redes cableadas, pero esto no permite la recepción portátil. Por otro lado, las transmisiones vía satélite con contenidos locales o regionales no siempre son viables económicamente. DVB-T permite utilizar dos tipos diferentes de redes: redes de frecuencia múltiple (MFN) o redes de frecuencia única (SFN) [1]. En una MFN todos los transmisores utilizan distintas frecuencias de transmisión, esto hace posible que dos transmisores adyacentes no empleen las mismas frecuencias. Este tipo de redes permiten la transmisión tanto de servicios globales como locales. La desventaja de esta topología es que es muy poco eficiente en su uso del espectro.

Por otro lado en una red SFN todos los transmisores están sincronizados, esto es, utilizan las mismas frecuencias y transmiten los mismos contenidos. Esta topología cuenta con diversas ventajas entre las que se puede destacar: mejor cobertura, potencia de transmisión menor, mayor fiabilidad y un uso eficiente del espectro. Sin embargo, como las señales transmitidas por los transmisores tienen que ser exactamente iguales, este tipo de redes no permite la provisión de servicios locales.

En este artículo se va analizar el uso de la modulación jerárquica y del time slicing para compatibilizar la provisión de servicios globales y locales en redes SFN. El uso la modulación jerárquica para proveer servicios locales en una red MFN ya ha sido considerado anteriormente [2]. Nosotros proponemos usar la modulación jerárquica como un mecanismo eficiente para proveer servicios locales en el flujo de baja prioridad (LP) y servicios globales en el flujo de alta prioridad (HP) con todas las ventajas de una red SFN. El otro enfoque propuesto en este artículo se basa en la utilización de la técnica utilizada en DVB-H llamada time slicing [3]. Mediante esta técnica se divide el tiempo en slots temporales, dedicando algunos a la transmisión de servicios globales y los otros a la transmisión de servicios locales. La modulación jerárquica ya se incluyó en el estándar DVB-T [1]. El time slicing es retro-compatible ya que los receptores existentes pueden seguir utilizándose sin modificar su hardware.

Las prestaciones de estas soluciones son evaluadas mediante simulaciones en un canal Rice (típicamente utilizado para recepción fija) para ambos tipos de servicios, globales y locales. La aplicación de estas soluciones en términos de propagación y cobertura también han sido analizadas en un escenario real.

El resto del artículo se estructura de la siguiente manera: en la sección II se explican las diferentes soluciones técnicas y las topologías de red propuestas para la provisión de servicios locales y globales en redes SFN. En la sección III se discuten algunos aspectos de implementación de dichas soluciones. La metodología para la estimación d cobertura de las LSA y GSA es presentada en la sección IV. La sección V presenta los 


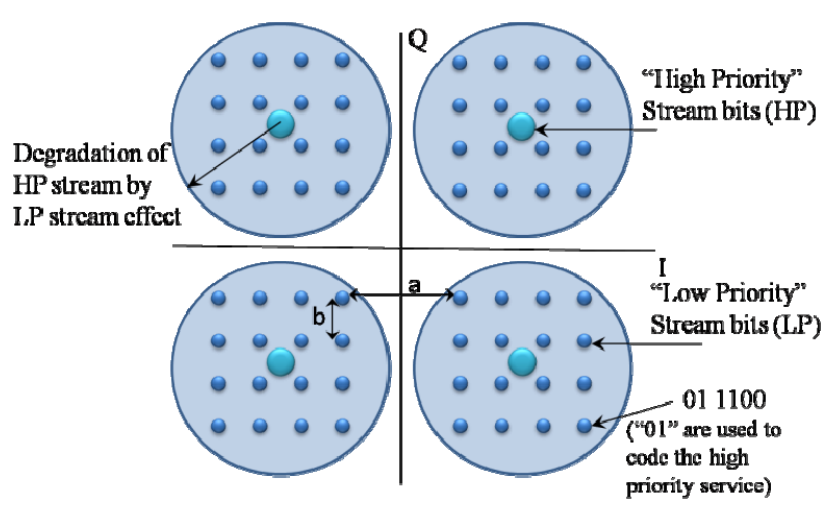

Figura 1. Ejemplo de Modulación Jerárquica.

principales resultados de esta investigación. Finalmente en la sección VI se presenta un ejemplo de estimación de cobertura para los servicios globales y locales en la red DVB-T de la región de Valencia (España).

\section{SOLUCIONES TÉCNICAS PARA LA PROVISIÓN DE SERVICIOS GLOBALES Y LOCALES EN REDES DVB-T SFN.}

\section{A. Modulación Jerárquica}

La modulación jerárquica es un modo de transmisión incluido en el estándar DVB-T que permite la transmisión de dos flujos TS en un único canal RF, con la particularidad de que cada uno de estos flujos TS es protegido de forma asimétrica. El primer flujo, al que llamamos flujo de alta prioridad (en adelante HP), es mapeado en una QPSK y define el cuadrante del símbolo transmitido. En la Fig. 1 se muestra un ejemplo, si la secuencia de bits que queremos transmitir en el flujo LP es '01', el cuadrante elegido será el derecho inferior. El otro flujo se llama flujo de baja prioridad (LP), puede modularse en una 16QAM o en una 64QAM y define la posición del símbolo dentro del cuadrante marcado por el flujo HP. En el ejemplo de la Fig. 1 la secuencia de bits del símbolo LP '1100' define la posición dentro del cuadrante.

Adicionalmente, en la modulación jerárquica se utiliza un parámetro llamado $\alpha$ para caracterizar el sistema. El parámetro $\alpha$ se define como la distancia mínima entre 2 símbolos de distinto cuadrante ( $a$ en la Fig. 1) dividido entre la distancia mínima entre 2 símbolos del mismo cuadrante $(b)$.

$\mathrm{Si}$ se usa la modulación jerárquica un receptor con bajo nivel de señal (causado por la distancia al transmisor, fading, interferencias o ruido impulsivo) sólo sería capaz de detectar el cuadrante del símbolo recibido y por lo tanto la información del flujo HP podría decodificarse pero no la del flujo LP, sin embargo un receptor con un nivel adecuado de señal sería capaz de decodifica la información de ambos flujos, HP y LP.

\section{1) Servicios locales usando modulación jerárquica}

Es importante señalar que la modulación jerárquica no establece ninguna limitación en cuanto a que tipos de datos son transmitidos por cada uno de los flujos, de hecho la información transportada por cada uno de los flujos no tiene por qué estar relacionada. Por lo tanto, es posible transmitir contenidos globales y locales mediante la modulación jerárquica, utilizando el flujo HP para los contenidos globales debido a que ellos requieren mayor cobertura, y el flujo LP para los contenidos locales. La modulación jerárquica

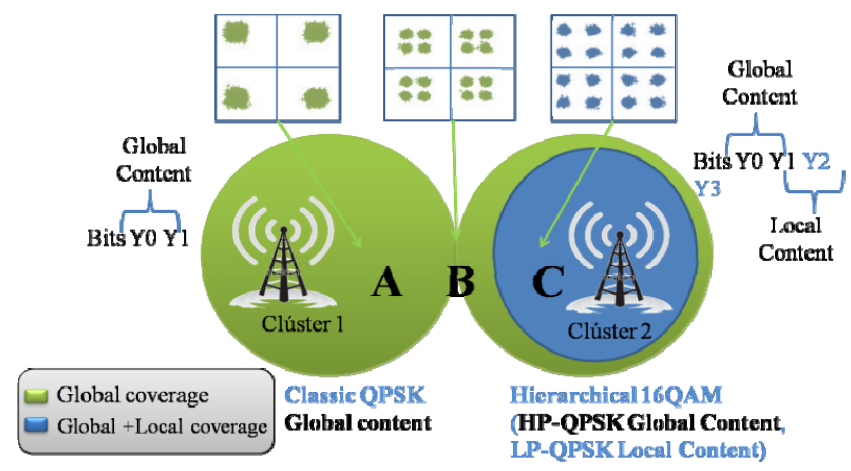

Figura 2. Topología SFN utilizando modulación jerárquica con contenidos locales en el flujo LP en uno de los transmisores.

convierte las celdas QAM de un orden inferior a un orden superior, por ejemplo pasa de una QPSK(global) a una 16QAM (global + local) o a una 64QAM (global + local). En cada uno de estos casos la modulación jerárquica proporciona 2 o 4 bits adicionales por celda y símbolo, respectivamente, para servicios locales. Dependiendo de la necesidad o no por parte del transmisor de emitir contenidos locales, podemos distinguir las siguientes dos topologías.

a) Algunos transmisores emiten contenidos locales

En esta topología SFN no todos los transmisores tienen contenidos locales que transmitir y por lo tanto se podría dar el caso en el que un transmisor sólo transmite contenidos globales y el transmisor adyacente transmite globales y locales. Los transmisores sin contenidos locales, deben usar el mismo esquema de transmisión del flujo HP que usan los demás transmisores para poder conseguir una combinación constructiva de los bits de este flujo.

La Fig. 2 muestra un ejemplo donde un transmisor se configura con una 16QAM jerárquica (contenidos globales y locales) y el otro transmisor se configura con una QPSK (global). En la figura se pueden distinguir 3 zonas de cobertura diferentes (A, B y C). Un receptor situado en la zona A puede decodificar solo los contenidos globales, porque la señal recibida es básicamente una constelación QPSK. En la zona $\mathrm{B}$ es posible recibir los contenidos globales a través de la combinación de las señales transmitidas por ambos transmisores (ganancia SFN). Por último un receptor situado en la zona $\mathrm{C}$ será capaz de recibir tanto los contenidos globales como locales ya que la modulación 16QAM jerárquica llega con una potencia elevada.

\section{b) Todos los transmisores emiten contenidos locales}

La otra posibilidad es que todos los transmisores emitan contenidos globales y locales simultáneamente, como se muestra en la Fig. 3. Las zonas A, B y C son áreas de servicios locales (LSA) y los contenidos locales a transmitir en cada una de ellas son diferentes. Esto provoca que los flujos LP de transmisores de distintas LSA provoquen interferencias entre ellos. Un receptor situado en cualquiera de estas zonas sería capaz de decodificar tanto los contenidos globales como locales, ya que al estar próximo al transmisor reciben la señal con un SINR elevada. Sin embargo un receptor situado en la zona D no tendrá suficiente SINR para decodificar los contenidos locales, recordar que dichos contenidos se combinan destructivamente, pero sí para decodificar los 


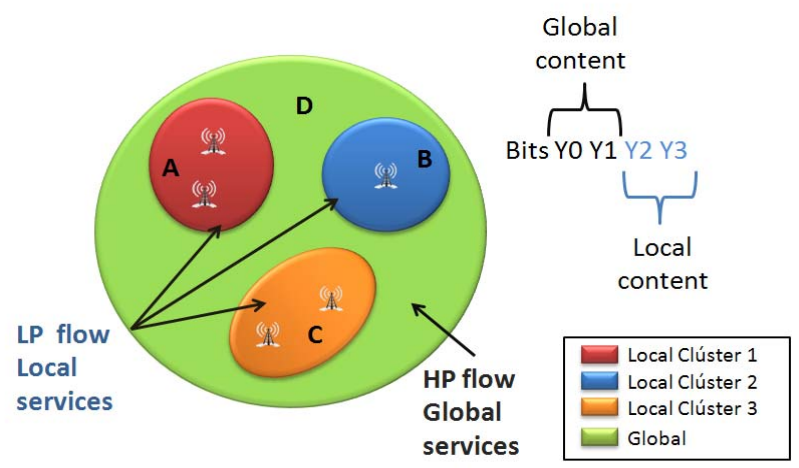

Figura 3. Topologia de red SFN utilizando modulacion jerarquica en todos los transmisores para emitir contenidos locales y globales.

contenidos globales, ya que los flujos HP se combinan constructivamente. De hecho los contenidos globales se reciben con todas las ventajas que proporciona la topología SFN.

\section{B. Time Slicing}

La otra tecnología que se propone para proveer servicios locales en redes de frecuencia única es el Time Slicing [4]. El time slicing es una técnica ya empleada en otros estándares como por ejemplo DVB-H, que consiste en la multiplexación temporal de los servicios que se van a transmitir.

El envío de cada uno de estos servicios se hace utilizando todo el ancho de banda que proporciona el canal durante un periodo de tiempo determinado. Como el bitrate total es mayor que el que requiere el servicio, hay instantes de tiempo en los que no transmitiremos información del servicio en cuestión sino de otro. La técnica se basa en la transmisión a ráfagas.

\section{1) Servicios locales usando Time Slicing en DVB-T}

La trasmisión de servicios locales en una red SFN DVB-T utilizando la técnica del time slicing requiere definir 2 periodos de tiempo, uno dedicado a la transmisión de contenidos globales, y el otro a contenidos locales. La Fig. 4 muestra la distribución temporal de los servicios en una transmisión que utiliza time slicing. En general, podemos distinguir 2 casos o situaciones:

\section{a) Periodo contenidos globales}

Durante este periodo todos los transmisores de la red transmiten los mismos contenidos, ya que son comunes, de forma sincronizada. Las señales transmitidas durante este periodo se encuentran con un escenario SFN y por lo tanto poseen las ventajas propias de estas redes como una mayor cobertura, menor infraestructura y potencia requerida, uso más eficiente del espectro, etc.

\section{b) Periodo contenidos locales}

Durante este periodo cada uno de los transmisores emite los contenidos locales que corresponden según la LSA donde se encuentren. Esto provoca que la topología, durante estos periodos, sea la de una red en la que existe interferencia entre transmisores adyacentes. La consecuencia de esto es que la cobertura de los contenidos transmitidos durante este periodo se ve afectada por las interferencias y por lo tanto se reduce. Un receptor situado cerca de un transmisor será capaz de

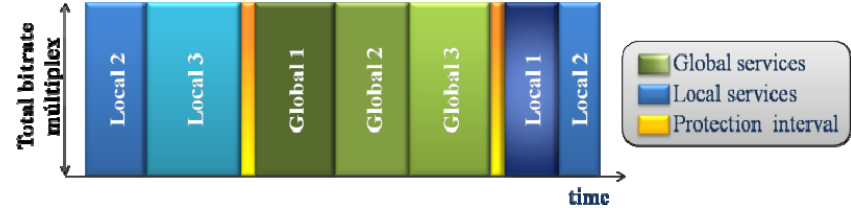

Figura 4. Técnica del time slicing.

decodificar tanto los contenidos globales como locales, ya que la señal de dicho transmisor predomina sobre la señal recibida de otros transmisores. Sin embargo, un receptor situado lejos de los transmisores sólo será capaz de decodificar los contenidos globales, ya que los locales sufren interferencias.

La capa física del estándar DVB-T entrelaza la información con el objetivo de protegerla de los errores que se producen en el canal de transmisión. Para que el time slicing en DVB-T funcione y permita la provisión de servicios locales debe garantizarse que los contenidos locales y globales no se mezclen, por lo tanto requiere la introducción de un periodo de protección entre los contenidos globales y locales, y viceversa [4], como se explica en la sección III.B.

\section{Servicios locales usando modulación jerárquica y time slicing.}

Las soluciones técnicas propuestas para la provisión de servicios locales en redes SFN pueden ser combinadas con el objetivo de reducir la interferencia entre distintas LSA, o para aumentar el bitrate que puede dedicarse a los contenidos globales. Para esto en el presente artículo se proponen dos formas de combinar la modulación jerárquica con el time slicing.

1) Modulación jerárquica con time slicing en el flujo $L P$

En esta topología todos los clusters dentro de la SFN usan modulación jerárquica simultáneamente, el flujo HP lleva información de contenidos globales y el flujo LP lleva información de contenidos globales y locales multiplexada mediante la técnica del time slicing. Para esto, se definen dos slots temporales en el flujo LP, uno de ellos se dedica a transmitir contenidos globales y el otro slot a contenidos locales. Esto es muy útil cuando no hay suficientes contenidos locales para aprovechar todo el bitrate que proporciona el flujo LP. Esta propuesta dota al sistema de mucha flexibilidad, ya que permite la elección de qué porcentaje del flujo LP se dedica a cada tipo de servicios.

El uso del time slicing en el flujo LP para transportar contenidos globales provoca que se formen dos áreas de cobertura diferentes para este tipo de contenidos, como se muestra en la Fig. 5. Por un lado tenemos la zona de cobertura para los contenidos globales transportados en el flujo HP (zona A) y por otro lado la cobertura de los contenidos globales transportados en el flujo LP (zona C). Una tercera área de cobertura (Zona $\mathrm{B}$ ) estará cubierta por los respectivos contenidos locales emitidos por cada clúster de la LSA. 


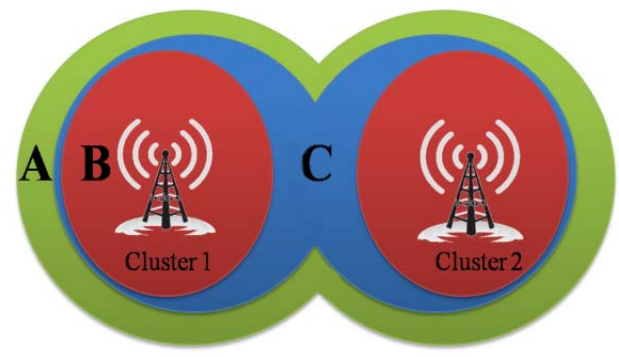

Global coverage (HP) $\square$ Global coverage (LP) $\square$ Local coverage (HP)

Figura 5 Áreas de cobertura obtenidas con modulación jerárquica y time slicing en el flujo LP.

Esta topología proporciona el escenario ideal para incorporar codificación de video escalable (SVC) de forma eficiente en los contenidos globales. SVC es un añadido al códec de video H.264/AVC cuyo objetivo es proveer de forma eficiente opciones de escalabilidad a contenidos codificados mediante H.264 [5]. SVC codifica la información en una capa base y en una o varias capas de mejora. En la topología de red utilizando modulación jerárquica y time slicing, la capa base del contenido global codificado mediante SVC se podría transportar en el flujo HP, ya que debe ser más robusta, y la capa de mejora se transportaría durante el slot dedicado a servicios globales dentro del flujo LP. De esta forma, un receptor situado en una zona con buen nivel de señal será capaz de decodificar ambas capas y reproducirá los contenidos con máxima calidad, sin embargo otros receptores situados en zonas con malas condiciones de recepción sólo serían capaces de decodificar la capa base, y por lo tanto de reproducir los contenidos con una calidad reducida.

\section{2) Modulación jerárquica con time slicing entre clusters} vecinos

Esta topología para combinar la modulación jerárquica y el time slicing, se diferencia de la topología anterior en la forma de emplear el time slicing sobre el flujo LP. Si dos o más clusters (o LSA) transmiten sus propios contenidos locales sobre el flujo LP simultáneamente y los transmisores de dichos clusters están cercanos entre ellos se crean interferencias que provocan que las áreas de cobertura de los respectivos contenidos locales sean reducidas. Con el objetivo de reducir este efecto, esta topología realiza un multiplexado temporal del flujo LP entre las distintas LSA que forman la red.

Durante un slot de tiempo determinado, el transmisor emitirá con modulación jerárquica (flujo HP y LP), sin embargo durante los slots que no tiene asignado el transmisor emitirá constelación clásica equivalente al flujo HP de la jerárquica. Se puede reutilizar los slots cuando los transmisores que comparten dicho slot están lo suficientemente alejados, de forma que no se interfieren entre ellos. Es importante destacar que conviene utilizar el mínimo número de slots posibles ya que de esa forma la capacidad total de la red aumenta. La Fig. 6 muestra un ejemplo de red con 4 slots definidos para contenidos locales.

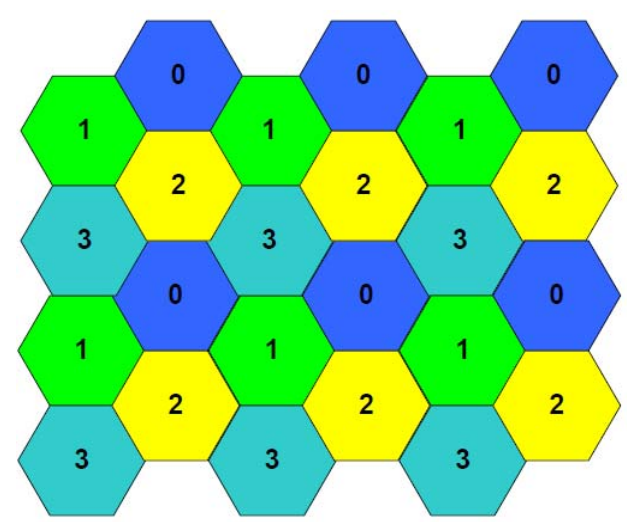

Figura 6. Esquema de reutilización de los slots de tiempo para la transmisión de contenidos locales en el flujo LP. (Flujo LP dividido en 4 slots).

\section{ASPECTOS DE IMPLEMENTACIÓN}

\section{A. Señalización TPS con Modulación jerárquica}

La señalización TPS (Transmission Parameter Signaling) [1] se ve afectada por el hecho de que un transmisor emita en modo jerárquico y uno cercano no. Para solucionar esto y garantizar la decodificación de los contenidos globales en sus respectivas áreas de interés, es necesario señalizar que todos los transmisores están emitiendo en modo jerárquico, incluyendo aquellos que en realidad no lo están haciendo. En estas zonas sin emisión de contenidos locales, el receptor recibirá el contenido como si fuera en el flujo HP de una modulación jerárquica.

\section{B. Intervalo de adaptación con time slicing}

Al usar el time slicing es necesario garantizar que los globales locales y globales no se mezclen debido a los entrelazados de capa física propia de DVB-T [1]. Para conseguir esto es necesaria la introducción de intervalos de protección, cuyo tamaño depende de varios parámetros como el tamaño de la FFT, la constelación y el code-rate utilizado. Los bloques funcionales de la cadena de transmisión DVB-T a tener en cuenta en el cálculo del intervalo de protección son: el entrelazador externo, entrelazador interno y el codificador interno. La ecuación (1) define el tamaño necesario de los intervalos de adaptación en función de los parámetros de configuración de dichos bloques.

$$
T=\frac{v^{*} n * 126 * C R}{8 * 204}+11
$$

Donde $v$ es el tamaño de palabra utilizado y depende de la constelación elegida, $v$ vale 2 en una QPSK, 4 en una 16QAM y 6 en una 64QAM. El parámetro $n$ es el número de bloques de 126 palabras que cabe en un símbolo OFDM, 12 bloques para una de $2 \mathrm{~K}$ o 48 bloques para una FFT de $8 \mathrm{~K}$. Y por último, CR es el valor del factor de expansión debido al code rate utilizado en el codificador interno.

A continuación se calcula el tamaño del intervalo de guarda para el modo de transmisión utilizado actualmente en España (8K, 64QAM, CR 2/3, GI 1/4). Los distintos parámetros toman los siguientes valores: $v=6 ; n=48 ; C R=2 / 3$. . 


$$
\frac{6 * 48 * 126 * 0.66}{8 * 204}+11=25.862 \rightarrow 26 \text { paquetes TS }
$$

\section{Señalización PSI/SI}

Las tablas PSI/SI (Program Specific Information/ Service Information) tienen la función de que el receptor pueda identificar que contenidos o programas lleva un determinado flujo TS y como acceder a ellos [6]. Por lo tanto para que un receptor consiga acceder a los contenidos deberá poder decodificar correctamente la información de las tablas PSI-SI. Para conseguir esto, es necesario que la información que viaje en las tablas sea idéntica en todos los transmisores. De forma que un receptor situado en las zonas de interferencia pueda decodificar la información de las tablas PSI/SI. La información relativa a los contenidos locales propios de cada LSA, se transmiten en la LSA correspondiente dentro de un servicio local del tipo guía electrónica de programación (EPG).

\section{METODOLOGÍA PARA LA ESTIMACIÓN DE COBERTURA}

La estimación de cobertura de las LSA y GSA para recepción fija se define como aquellas áreas donde la $\operatorname{SINR}$ (señal a interferencia más ruido) supera cierto umbral. La ecuación (2) muestra el valor de SINR en función de varios parámetros [7].

$$
\operatorname{SINR}=\frac{P_{c}}{P_{I}+I+P_{N}}
$$

El valor $P_{C}$ define la suma de las potencias recibidas de los distintos transmisores de la LSA y es calculado mediante la ecuación (3). La señal recibida más fuerte se considera como la principal y las otras señales recibidas de la propia LSA se consideran ecos artificiales.

$$
P_{c}=\sum_{i \in L S A} P_{r i} * W\left(t_{i}\right)
$$

Dependiendo del retardo $(\Delta t)$ relativo entre los ecos y la señal principal, estos ecos pueden contribuir o interferir en la señal que llega al receptor. Esta contribución se define con las funciones de peso $\mathrm{W}(\Delta \mathrm{t})$ especificadas en la ecuación (4).

$$
W(\Delta t)= \begin{cases}0, & \text { if } \Delta t \leq T_{g}-T_{u} / 3 \\ \left(\frac{T_{u}+\Delta t}{T_{u}}\right)^{2}, & \text { if } T_{g}-T_{u} / 3<\Delta t \leq 0 \\ 1, & \text { if } 0 \leq \Delta t \leq T_{g} \\ \left(\frac{T_{u}+T_{g}-\Delta t}{T_{u}}\right)^{2}, & \text { if } T_{g}<\Delta t<T_{u} / 3 \\ 0, & \text { if } T_{u} / 3<T_{g}\end{cases}
$$

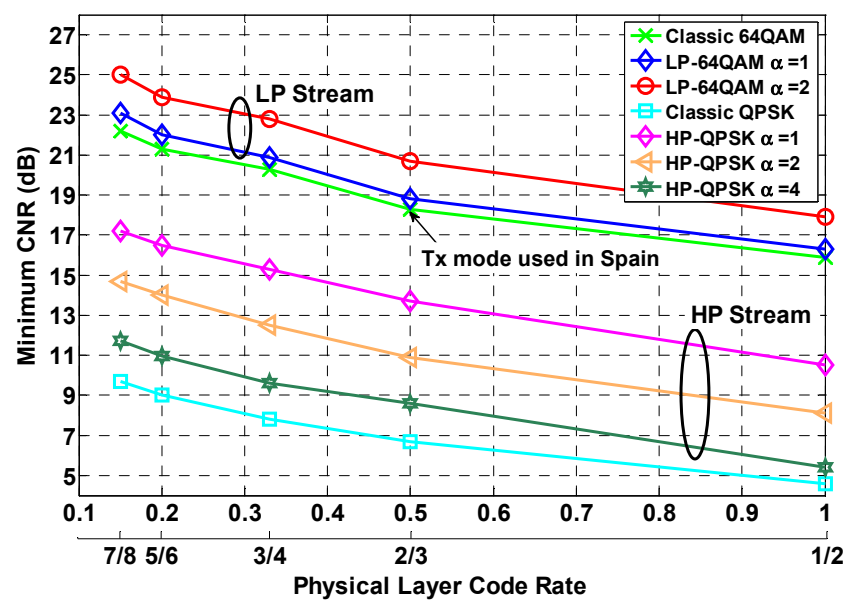

Figura 7. Rendimiento de la modulación jerárquica en canal Rice.

Donde $T u$ es el periodo útil del símbolo OFDM y $T g$ es el intervalo de guarda. Por otro lado, las señales interferentes que tienen su origen en la misma LSA, llamas $P_{I}$ en la ecuación 2, se calculan mediante la ecuación (5).

$$
P_{c}=\sum_{i \in L S A} P_{r i} *\left(1-W\left(t_{i}\right)\right)
$$

Por último, el término $I$ de la ecuación 2 representa la suma de las señales recibidas de todos los transmisores que no pertenecen a la LSA analizada y $P_{N}$ es el nivel de ruido recibido.

Para calcular el SINR de las GSA se usa la misma ecuación (2) que utilizamos para las LSA, sin embargo cambia la forma de calcular cada uno de los términos. El término $P_{C}$ representa la suma de las potencias $P r$ recibidas de todos los transmisores de la SFN. El termino $P_{I}$ representa la suma de todas las originadas en la propia red SFN pero que debido al retardo $(\Delta t)$ con el que llegan al receptor interfieren. El término $I$ es la suma de las señales interferentes recibidas cuyo origen no es un transmisor de la propia red SFN y $P_{N}$ es la potencia de ruido.

\section{RESULTADOS Y DISCUSIONES}

\section{A. Pruebas de laboratorio de la modulación jerárquica para receptores fijos.}

La Fig. 7 muestra la CNR requerida para una recepción Quasi Error Free (QEF) (Una recepción es QEF cuando la BER es menor que $2 * 10^{-4}$ después de Viterbi, esto se corresponde con una BER de $2 * 10^{-11}$ después de Reed Solomon. Un BER of $2 * 10^{-11}$ significa 1 bit erróneo cada 5 horas a una tasa de $5 \mathrm{Mbit} / \mathrm{s}$.) en un canal Ricean para distintas constelaciones, parámetros $\alpha$ y code rates. Estas curvas han sido obtenidas mediante medidas de laboratorio en un receptor profesional de DVB-T. De acuerdo con los resultados un receptor fijo en España requiere aproximadamente una CNR de $18 \mathrm{~dB}$ para tener una recepción QEF con el actual modo de transmisión (64QAM no jerárquico, $8 \mathrm{~K}, \mathrm{CR} 2 / 3$, GI 1/4). Utilizando modulación jerárquica y $\alpha=1$, la degradación del LP es sólo de $0.5 \mathrm{~dB}$ con lo que obtiene casi los mismos niveles de cobertura que la 64QAM clásica. Además el flujo $\mathrm{HP}$ requiere casi $4 \mathrm{~dB}$ menos que la $64 \mathrm{QAM}$ no jerárquica incrementando la cobertura de los contenidos globales. Para $\alpha$ $=2$, la degradación del LP aumenta hasta $2 \mathrm{~dB}$ y la ganancia 


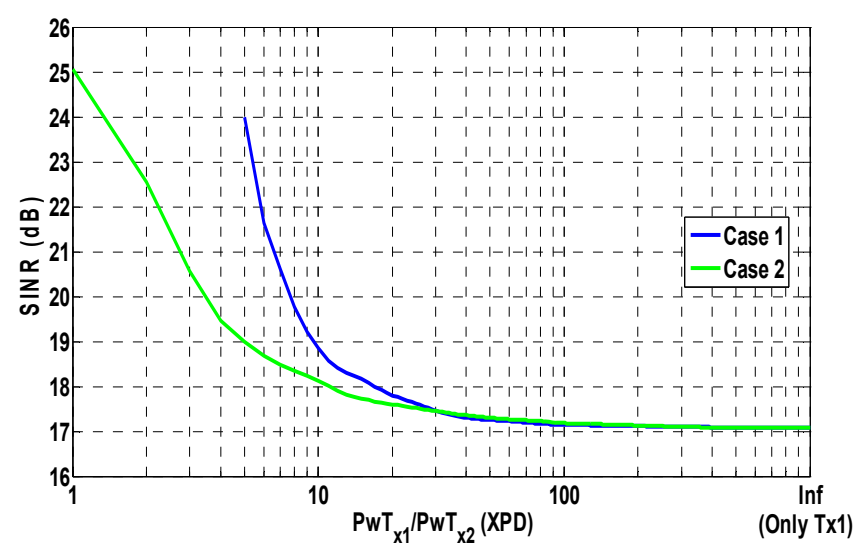

Figura 8. SINR para recepción QEF en función de la XPD para servicios locales en canal Rice usando la modulación jerárquica.

del HP aumenta hasta $7 \mathrm{~dB}$. Para $\alpha=4$, el rendimiento del flujo LP es muy pobre (no se muestra en la figura) y el rendimiento del HP está cerca del de una QPSK clásica (sólo 1 $\mathrm{dB}$ por debajo).

\section{B. Rendimiento de la modulación jerárquica para la transmisión de contenidos locales y globales}

La Fig. 8 representa la mínima CNR requerida para una recepción QEF de los contenidos globales (flujo HP) en función de la relación de potencias recibidas de los transmisores PwT1/PwT2 (XPD). Las curvas de rendimiento han sido obtenidas mediante simulaciones de la recepción de una señal DVB-T emitida desde dos transmisores diferentes. Cada transmisor se ha simulado con FFT $8 \mathrm{~K}$, GI $1 / 4$ y QPSK CR 2/3 si sólo transmite contenidos globales o con una modulación jerárquica 64QAM, $\alpha=1, \mathrm{CR} 2 / 3$ (para HP y LP) si transmite contenidos globales y locales. Con el fin de obtener el rendimiento sin tener en cuenta la influencia de la potencia total transmitida, ésta se normaliza a 1.

Las curvas representan el rendimiento para la recepción de la información emitida desde el transmisor 1 en los siguientes 3 casos. Caso 1 (curva azul), ambos transmisores usan modulación jerárquica para transmitir contenidos globales (iguales) y locales (diferentes); caso 2 (curva verde), el transmisor 1 usa modulación jerárquica y el transmisor 2 usa una QPSK clásica ya que sólo transmite contenidos globales; caso 3 (curva roja), el transmisor 1 solo transmite contenidos globales en una QPSK y el transmisor 2 transmite contenidos globales y locales con modulación jerárquica.

En el caso 1, un receptor fijo requiere $11.3 \mathrm{~dB}$ de CNR para una recepción QEF cuando el transmisor 1 es claramente dominante y $9.1 \mathrm{~dB}(-2.2 \mathrm{~dB})$ cuando la potencia recibida de los dos transmisores, transmisor $1 \mathrm{y}$ transmisor 2 , es comparable. Este efecto es causado por la combinación de los flujos LP, que son diferentes y provocan que los símbolos en cada cuadrante se desplacen hacia el centro del cuadrante correspondiente. En el caso 2 se aprecia un comportamiento parecido aunque la reducción en este caso es de hasta $4.5 \mathrm{~dB}$ debido a que el transmisor 2 utiliza una QPSK clásica y aún obliga más a los símbolos a desplazarse hacia el centro del cuadrante. En el caso 3 la principal señal es una QPSK y se penaliza con hasta $1.5 \mathrm{~dB}$ de CNR por efecto del flujo LP del transmisor 2 cuando las señales recibidas de ambos tienen la misma potencia.

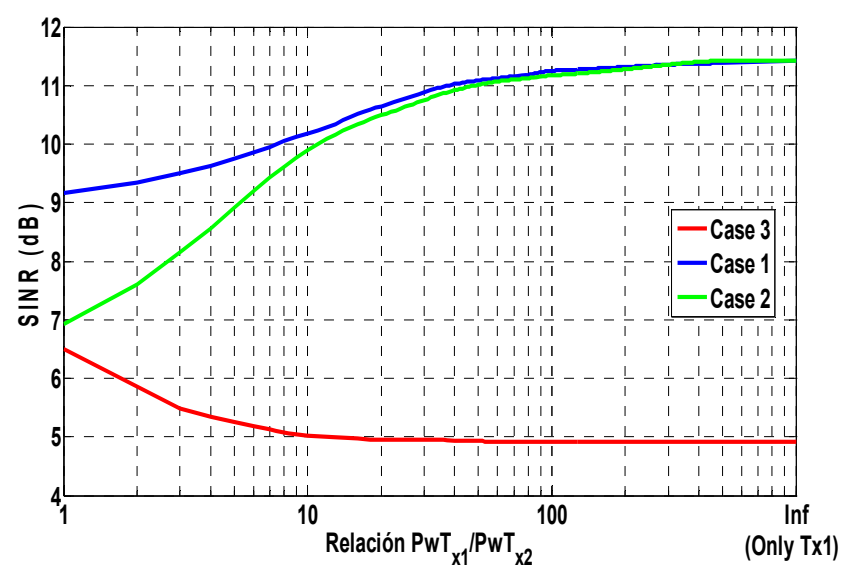

Figura 9. SINR para recepción QEF en función de la XPD para servicios globales en canal Rice usando la modulación jerárquica.

El rendimiento de los servicios locales transmitidos en el flujo LP se muestra en la Fig. 9. Para el caso 1, un receptor requiere $17 \mathrm{~dB}$ para una recepción óptima de los contenidos locales. Cuanto mayor sea la interferencia producida por el transmisor 2, mayor es el nivel de CNR requerido para una buena recepción. Se puede ver que para valores de XPD en torno a 5 el nivel de interferencias es tan elevado que es imposible decodificar los contenidos locales. En el caso 2, la señal principal es jerárquica y la del transmisor secundario es una QPSK que sólo contiene globales.

La señal del transmisor 2 obliga a los símbolos a desplazarse hacia el centro del cuadrante afectando a la decodificación del flujo LP. En general, las interferencias producidas por otros flujos LP reducen la cobertura de los contenidos locales y hay áreas en las que no es posible la recepción de los contenidos locales. Sin embargo esto no suele ser un problema ya que estas áreas normalmente están alejadas del transmisor y solo requieren recibir contenidos globales.

\section{Evaluación del rendimiento del time slicing}

Al usar la técnica del time slicing para la provisión de servicios locales, se han definido 2 slots temporales por ciclo. En el primer slot todos los transmisores transmiten la misma información (información global) y por ello durante este slot temporal hay ganancia SFN en área de cobertura. Asumiendo que todas las señales se considerarán que llegan dentro del intervalo de guarda y perfectamente sincronizadas el receptor no sufrirá interferencias y todas las señales se combinarán constructivamente. Los 2 transmisores han sido simulados con el modo de transmisión usado en España (64QAM, 8K, CR 2/3, GI 1/4) y los resultados obtenidos se muestran en la Fig.10. Como puede verse el minino CNR requerido para decodificar los servicios globales es $16 \mathrm{~dB}$. Este valor es constante e independiente del valor de la XPD (recordar que normalizamos la potencia total). Durante el otro slot temporal cada transmisor emite los contenidos locales propios de la LSA donde se encuentra, en este caso las señales de los otros transmisores son consideradas interferencias.

Las curvas de rendimiento de los contenidos locales en unc anal Rice y Rayleigh también son indicadas en la Fig. 10. Sin las interferencias provocadas por el transmisor 2, un receptor en canal Rice requiere $16 \mathrm{~dB}$ para decodificar los contenidos locales del transmisor 1 . Sin embargo para relaciones de 


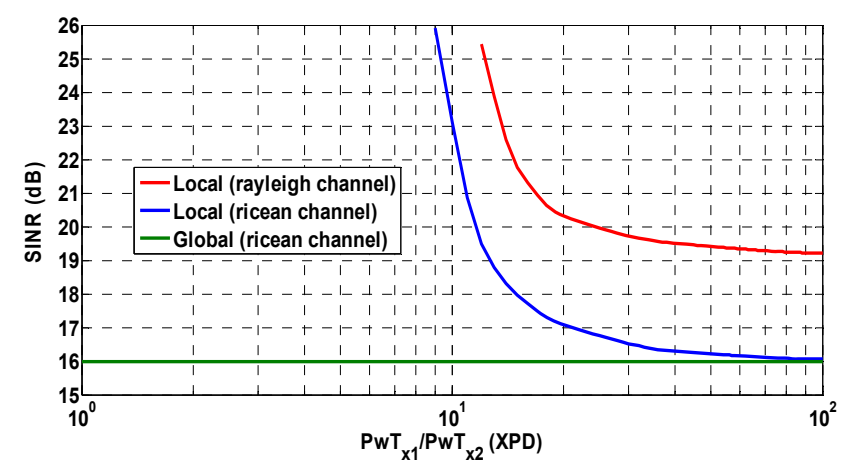

Figura 10. SINR requerida para la recepcion de contenidos locales y globales en un canal Rice utilizando tecnicas de time slicing en una red SFN. Modo de transmisión 64QAM, FFT 8K, CR 2/3 y GI 1/4.

potencia (XPD) menores de 10 las interferencias son tan altas que impiden la decodificación de los contenidos locales.

\section{Tamaño del tiempo de ciclo en el time slicing}

La introducción de un periodo de protección durante el cual se introducen paquetes null produce una pérdida de eficiencia en términos de bit rate total que es capaz de transportar el múltiplex. El número de paquetes a introducir en los intervalos de guarda depende del modo de transmisión empleado, pero es constante con respecto al tamaño del tiempo de ciclo del sistema. El tiempo de ciclo es el tiempo que transcurre entre ráfagas del mismo tipo de contenidos. Para tiempos de ciclo pequeños la proporción entre datos útiles e información de relleno es más pequeña, menor eficiencia, y para tiempos de ciclos mayores pasa lo contrario, mayor eficiencia.

La Fig.11 muestra la eficiencia del multiplex en función del tiempo de ciclo para distintos modos de transmisión. Se puede apreciar que para el modo de transmisión QPSK 2/3 con valores de tiempo de ciclo por encima de 0.8 segundos el overhead ya se encuentra por debajo del $1 \%$, para los modos 16QAM y 64QAM esta situación se da con tiempos de ciclo de 0.5 y 0.4 segundos respectivamente. Además de la eficiencia del múltiplex el tiempos de ciclo influye en el tamaño de los buffers que debe tener el receptor, para ciclos mas grandes el receptor necesitará buffers y memorias más grandes. Sin embargo, los test de laboratorio que hemos llevado a cabo durante la realización de este artículo que tiempos de ciclo menores que 1 segundo son admisibles por

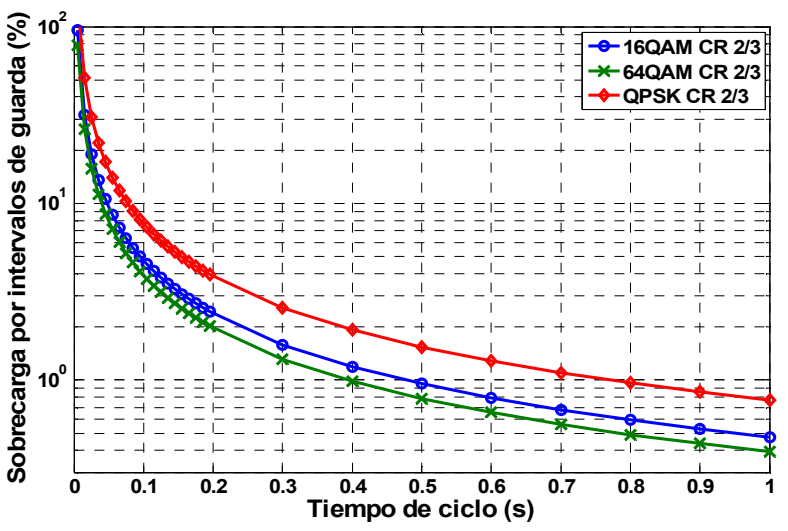

Figura 11. Porcentaje de bit rate dedicado a los intervalos de guarda en función del tiempo de ciclo y del modo de transmisión

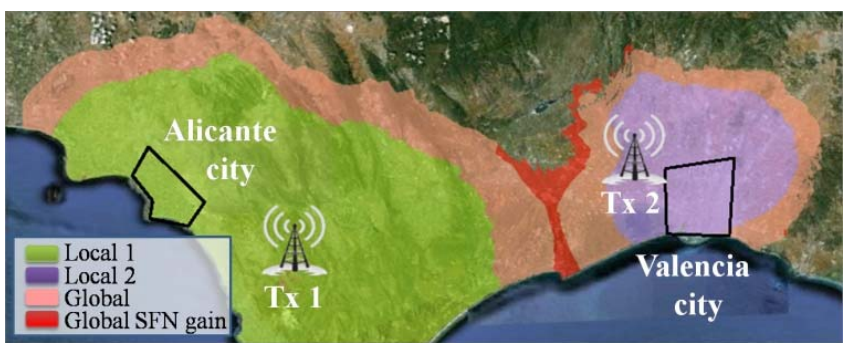

Figura 12. Área de cobertura para servicios globales y locales obtenidos con la solución de modulación jerárquica para redes SFN.

los receptores comerciales.

TABLA I. CONFIGURACIÓN DEL ESCENARIO REAL SIMULADO

\begin{tabular}{|c|c|c|}
\hline Ciudad & Alicante & Valencia \\
\hline Localización & $\begin{array}{c}38^{\circ} 38^{\prime} 45.30^{\prime \prime} \mathrm{N}, \\
0^{\circ} 19^{\prime} 47.26^{\prime \prime} \mathrm{W}\end{array}$ & $\begin{array}{c}39^{\circ} 19^{\prime} 44.06^{\prime \prime} \mathrm{N}, \\
0^{\circ} 32^{\prime} 12.29^{\prime \prime} \mathrm{W} .\end{array}$ \\
\hline EIRP & $5 \mathrm{Kw}$ & $4 \mathrm{Kw}$ \\
\hline Altura antena & $40 \mathrm{~m}$ & $50 \mathrm{~m}$ \\
\hline Frecuencia & $770 \mathrm{Mhz}$ & $770 \mathrm{Mhz}$ \\
\hline $\begin{array}{c}\text { Modo de } \\
\text { transmisión }\end{array}$ & $\begin{array}{c}\text { 64QAM jerárquica, } 8 \mathrm{k}, \\
\text { GI }, \mathrm{CR} 2 / 3(\text { tanto LP } \\
\text { como HP) }\end{array}$ & $\begin{array}{c}\text { 64QAM jerárquica, } 8 \mathrm{k}, \\
\text { GI }, \mathrm{CR} 2 / 3(\text { tanto LP } \\
\text { como HP) }\end{array}$ \\
\hline $\begin{array}{c}\text { Ganancia de } \\
\text { antena del } \\
\text { transmisor }\end{array}$ & $10 \mathrm{~dB}$ & $10 \mathrm{~dB}$ \\
\hline \multicolumn{2}{|c}{} & \\
\hline
\end{tabular}

\section{ESTIMACIÓN DE COBERTURA DE LOS DISTINTOS SERVICIOS EN UN ESCENARIO REAL}

La red TDT simulada está formada por dos LSA situadas en la Comunidad Valenciana (España), una en la ciudad de Alicante y el otro en la ciudad de Valencia. La tabla I muestra la configuración del escenario simulado y de los transmisores. Estos transmisores están separados $89 \mathrm{~km}$ y actualmente transmiten contenidos DVB-T. La simulación utiliza como modelo de propagación el modelo de Okumura-Hata extendido [8]. El área de cobertura de determina para recepción en exteriores con antenas en tejados y usando modulación jerárquica para transmitir contenidos globales y locales.

Por el lado del receptor se ha asumido una figura de ruido de 6 $\mathrm{dB}$, una ganancia de antena de $14 \mathrm{~dB}$, una desviación estándar de desvanecimiento de $5.5 \mathrm{~dB}$ y un margen de desvanecimiento de 9 dB. La Fig. 12 muestra la cobertura resultante para ambos tipos de contenidos. Los contenidos globales tienen una cobertura total en ambas ciudades (área color rosa) y una cobertura adicional en áreas rurales debido a la ganancia SFN de la red (zona rojo oscuro). Los contenidos locales están limitados a sus respectivas zonas y su radio de cobertura es de unos $55 \mathrm{~km}$ para el transmisor de Alicante (área verde) y de $38 \mathrm{~km}$ para el transmisor de Valencia (área morada).

\section{CONCLUSIONES}

En este artículo se ha comprobado que la coexistencia de contenidos globales y locales con la topología de red SFN es posible si se usa la modulación jerárquica, el time slicing o ambas técnicas de forma combinada en una red DVB-T. Los resultados obtenidos en este trabajo muestran que el tamaño de las LSA está limitado por las interferencias producidas en la red. Por el 
contrario, los contenidos globales mantienen todas las ventajas de las redes SFN frente al enfoque MFN, como son la gran cobertura y la eficiencia espectral. También se ha demostrado que la cobertura estimada para los contenidos locales en las distintas propuestas es lo suficientemente grande como para aplicarse en redes DVB-T reales.

\section{REFERENCIAS}

[1] ETSI TR 101190 v1.3.1, "Implementation Guidelines for DVB Terrestrial Services; Transmission Aspects,” Nov. 2008.

[2] H. Jiang and P.Wilford, "A hierarchical modulation for upgrading digital broadcast systems," IEEE Transactions on Broadcasting, vol. 51, no. 2, pp. 223-229, Jun. 2005.

[3] ETSI TR 102377 V1.4.1 “ Implementation guidelines for DVB handheld services", Jun 2009.

[4] G. May, P. Unger, "A New Approach for Transmitting Local Content Within Digital Single Frequency Broadcast Networks," IEEE Transactions on Broadcasting, vol.53, no.4, pp.732-737, Dec. 2007.

[5] H. Schwarz, D. Marpe, T. Wiegand, "Overview of the Scalable Video Coding Extension of the H.264/AVC Standard," IEEE Trans. on Circuits and Systems for Video Technology, vol.17, no.9, pp.1103-1120, Sept. 2007.

[6] ETSI TS 101162 V1.2.1, "Allocation of Service Information (SI) codes for DVB systems" July 2009.

[7] A. Mattson, "Single Frequency Network in DTV", IEEE transactions on Broadcasting, vol. 51, no. 4, pp.413-422, Dec. 2005.

[8] Y. Okumura, E. Ohmori, T. Kawano, and K. Fukuda, "Field Strength and its Variability in VHF and UHF Land-Mobile Service," Rev. Elec. Comm. Lab., vol. 16, no. 9-10, pp. 825-873, 1968.

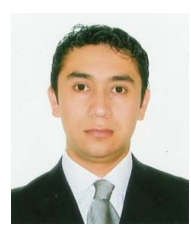

Jaime López Sánchez nació en Sogamoso, Boyacá Colombia, el 1 de Octubre de 1981. Se graduó de Ingeniero Electrónico en la Universidad Distrital Francisco José de Caldas-Bogotá en 2005. De 2005 a 2008 ejerció profesionalmente en el canal privado de televisión Caracol TV S.A, como ingeniero de emisión y transmisión. Recibió el título de Máster en Tecnologías redes y sistemas de comunicaciones de la Universidad Politécnica de ValenciaEspaña (UPV) en 2009. Actualmente es estudiante de Doctorado de la UPV e investigador del grupo de comunicaciones móviles del instituto iTEAM. Su investigación recibió el premio "Valencia Idea 2010" en la categoría TIC (Tecnologías de la Información y la Comunicación). Sus temas de interés son el estudio de nuevas soluciones técnicas en la evolución de los estándares DVB (Digital Video Broadcasting) para la provisión de servicios avanzados multimedia en redes de Televisión Digital, la planificación y optimización de estas redes para recepción fija, portable y móvil.

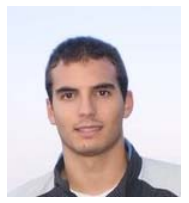

Carlos Romero Ruescas nació en Valencia-España en 1986. Recibió el título de ingeniero de telecomunicaciones en la Universidad Politécnica de Valencia (UPV) en 2010. Actualmente, trabaja con ingeniero R\&D en el grupo de comunicaciones móviles del instituto de Telecomunicaciones y Aplicaciones Multimedia iTEAMUPV. Sus temas de interés son la optimización de las actuales redes de Televisión Digital Terrestre TDT y el estudio y desarrollo de plataformas de encapsulación DVB-T2 y NGH.

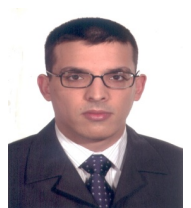

Dr. David Gómez-Barquero es investigador invitado post doctoral en el instituto Fraunhofer Heinrich Herzt (HHI) en Berlin-Alemania. Él es Doctor Ingeniero de Telecomunicación por la Universidad Politécnica de Valencia (España), y Máster en Telecomunicaciones por la Universidad Politécnica de Valencia y de la Universidad de Gävle (Suecia). Durante su formación doctoral realizó estancias como investigador invitado en la Universidad KTH de Estocolmo (Suecia), la Universidad de Turku (Finlandia), y la Universidad de Braunschweig (Alemania). También realizó prácticas en Ericsson Eurolab en Aachen (Alemania). Actualmente está realizando una estancia post-doctoral en el Instituto de Telecomunicaciones Fraunhofer HHI de Berlin, Alemania. Su principal área de interés es la radiodifusión de contenidos multimedia móviles tanto en sistemas DVB como en 3GPP MBMS.

El Dr. Gómez-Barquero dirige el grupo de especial interés de redes hibridas y de radiodifusión de la acción de cooperación europea COST2100 y fue coeditor del bluebook de DVB en mecanismos de corrección de errores en capas superiores UL-FEC como experto invitado. Actualmente, participa en el proceso de estandarización de la nueva generación de TV digital móvil DVBNGH.

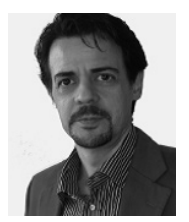

Dr. Narcís Cardona nació en 1963 en Barcelona-España. En 1990 Recibió el título de máster en telecomunicaciones de la Universidad Politécnica de Cataluña y en 1995 el título de Doctor en telecomunicaciones de la Universidad Politécnica de Valencia (UPV). Desde 1990 ha estado vinculado con la UPV donde actualmente es profesor catedrático y director del grupo de comunicaciones móviles. Adicionalmente es director del máster universitario en comunicaciones móviles y subdirector del Instituto de Telecomunicaciones y Aplicaciones Multimedia (iTEAM). El profesor Cardona ha liderado diferentes proyectos de investigación a nivel nacional y ha participado en proyectos europeos, redes de excelencia y otros foros de investigación, siempre en aspectos de comunicaciones móviles. Sus actuales áreas de interés incluye la caracterización del canal radio móvil, herramientas de planificación y optimización de sistemas celulares, técnicas RRM aplicadas a comunicaciones personalizadas y de radiodifusión en redes hibridas.

El profesor Cardona ha sido vicepresidente del COST273 y está actualmente a cargo del WG3 del COST2100 en el área redes de acceso vía radio. El fue presidente del la tercera conferencia internacional en sistemas de comunicaciones inalámbricas (ISWCS'06). 